total of $44(4.5 \%)$ had a positive CEA test ( $\geqslant 5 \mathrm{ng} / \mathrm{ml}$ plasma) at the outset. During the next four years six of them died of CEA-associated cancers, two were found to have colonic diverticula, and 15 were found to be heavy smokers. By contrast 18 of the 912 CEA-negative individuals developed CEA-associated cancers during this period. Re-examination four years later of 21 of the apparently healthy persons who had been CEA-positive in 1969 showed that two had occult cancers, one of colon and one of lung. This small yield of cancers does not justify extensive clinical study of asymptomatic CEApositive persons-which would have to be repeated-since half remain well for as long as four years and probably longer.

A simpler approach has been advocated by Lefall, who believes that annual testing of the faecal samples in the over 40 s could reduce the toll of colorectal cancer. ${ }^{5} \mathrm{His}$ idea is to issue the public with guaiac-impregnated paper slides, which they use for the test on three successive days after being on a highresidue meat-free diet. The slides are then returned to the doctor for development and reading. Lefall claims that this screening procedure yields a $5 \%$ positive rate and that $1 \%$ of these positives may be due to bowel cancer. His report did not state how the doctor decided whether to proceed to a formal examination of the bowel if the test was positive.

The simple occult blood test or the more complex annual medical check and proctoscopy would undoubtedly bring a crop of presymptomatic bowel cancers to the notice of the doctor and the patient. The crucial question is whether the lead time over the natural point of symptomatic presentation would result in a change in the mortality or only an extension of survival equal to the lead time.$^{6}$ At present this question is unanswered; as it can be resolved only by formal testing, the size of the problem should not be underrated. Maybe this is the type of research that the regional cancer organisations might co-ordinate, by comparing survival in screened and unscreened populations. Large numbers would be requiredthe incidence per 100000 at the age of 65-69 is 190 in men and 126 in women-so many general practitioners would need to be enlisted to help, and it is unlikely that there would be an answer in less than seven years. Such a programme might turn the tide of the disease; on the other hand such phrenetic activity with its extra demand for diagnostic facilities could hinder our ability to deliver health care for patients with overt disease-a nice point to debate. Maybe we should have been better off spending our time encouraging the patient with symptoms to seek advice and the general practitioner to keep the probability of bowel cancer well to the fore when dealing with the over-60s.

\footnotetext{
${ }^{1}$ Bussey, H J R, Dukes, C E, and Lockhart-Mummery, H E, in Cancer of the Rectum, ed C E Dukes, p 267. London, Livingstone, 1960.

${ }^{2}$ McSherry, C K, Cornell, G N, and Glenn, F, Annals of Surgery, 1969, $169,502$.

${ }^{3}$ Dales, L G, et al, Preventive Medicine, 1973, 2, 221.

4 Stevens, D P, Mackay, I R, and Cullen, K J, British fournal of Cancer, 1975, 32, 147.

5 Fournal of the American Medical Association, 1975, 234, 137.

${ }^{6}$ Zelen, M, and Feinleib, M, Biometrika, 1969, 56, 601.
}

\section{Cardiac contusion}

Contusion of the myocardium-recognisable damage to heart muscle short of rupture-is a fairly common concomitant of blunt injury to the chest, a type of injury that (until the oil crisis at least) has been steadily increasing around the world.
Nevertheless, while cardiac contusion is easily recognisable at necropsy from the obvious damage and bruising of the ventricles, it is a good deal more elusive clinically, both in its incidence and in its clinical definition.

Clinically, contusion is said to be present when, after blunt chest injury, there is electrocardiographic evidence of myocardial infarction or ischaemia which eventually disappears. Diagnosed by these criteria its incidence is increasing, owing to a general increase in such injuries and to heightened diagnostic awareness. Typically, there is chest pain of myocardial ischaemic type, but this may be difficult to elucidate since most patients have some radiological or clinical evidence of injury to the chest wall. The electrocardiographic changes are those of myocardial ischaemia and infarction, ${ }^{1}$ usually ST-segment and T-wave changes. These may not develop until two days after the injury, which may cause the diagnosis to be delayed or missed. Serum enzyme levels are often raised, but this is of no diagnostic value as the rise may be due to injuries of liver, lung, or skeletal muscle. Other diagnostic procedures such as measurements of cardiac output ${ }^{2}$ or technetium scans ${ }^{1}$ are too complex for most accident units.

Cardiac contusion is not usually fatal in itself: death may be due primarily to other injuries sustained, though sometimes contusion occurs with no external evidence of damage to the chest wall whatsoever. The specific dangers are the development of pericardial tamponade and dysrhythmias, including atrial fibrillation, supraventricular and ventricular tachycardia, and complete heart block. These tend to occur in patients who are suffering from hypotension and hypoxia and who are therefore the most seriously injured.

The importance of diagnosing, or at least suspecting, cardiac contusion in an injured patient lies in preventing fluid overload and starting cardiac monitoring to identify the dangerous dysrhythmias when they occur. Giving large volumes of fluid, particularly blood, is usual in the management of major blunt trauma, and overload may be fatal. In one survey ${ }^{1}$ four of the five patients with cardiac contusion who developed congestive cardiac failure did so during intravenous fluid replacement. If dysrhythmias are recognised quickly from the electrocardiogram and oscilloscope they can be managed effectively with, in the first instance, digitalis for supraventricular arrhythmias, lignocaine for ventricular tachycardia, and an intravenous pacemaker for heart block. Tamponade may be suspected from central venous and arterial monitoring and confirmed by injection of contrast medium into the right atrium, showing a wide shadow between it and the pericardium. It is treated by tapping with a needle or, better, a small catheter inserted under the xiphisternum by the Seldinger technique. Thoracotomy is rarely necessary for contusion alone. $^{3}$

Management of cardiac contusion does not end with discharge of the patient from the ward. Complications may occur months or even years later, ${ }^{45}$ and these may include ventricular septal defects, left ventricular aneurysms, or coronary artery occlusion. Suspicion of cardiac contusion should, therefore, be enough to trigger a cardiac rather than a purely orthopaedic approach to blunt trauma. Its recognition may prevent a relatively benign condition leading to fatal complications.

1 Jones, J W, Hewitt, R L, and Drapanas, T, Annals of Surgery, 1975, 181, 567.

2 Pomerantz, M, Delgado, F, and Eiseman, B, Surgery, 1971, 70, 865.

${ }^{3}$ McDermott, F T, and Douglas, M C, Australian and New Zealand fournal of Surgery, 1967, 37, 147.

${ }^{4}$ Doty, D B, et al, Annals of Surgery, 1974, 180, 452.

5 Stern, T, et al, fournal of the American Medical Association, 1974, 230, 1308 